IGCS20_1373

\section{CLEAR CELL ADENOCARCINOMA OF THE PERITONEUM ARISING ON A BACKGROUND OF ENDOMETRIOSIS - A RARE CASE}

${ }^{1} \mathrm{~K}$ Sherring*, ${ }^{1} \mathrm{~N}$ Wilkinson, ${ }^{2} \mathrm{P}$ Barton-Smith. 'University College London Hospitals NHS Foundation Trust, UK; ${ }^{2}$ Princess Grace Hospital, UK

\subsection{6/ijgc-2020-IGCS.300}

A 38 year old woman presented with a longstanding history of pain secondary to endometriosis. She had previously undergone major surgery with a segmental bowel resection seven years earlier. On assessment she was found to have a $3.7 \mathrm{~cm}$ right uterosacral ligament nodule with atypical appearances on transvaginal ultrasound scan. CA125 was 120 and risk of ovarian malignancy algorithm (ROMA) score was not suggestive of malignancy. A subsequent MRI scan, however, suggested suspicious findings. MDT discussion led to the decision to excise the mass along with a hysterectomy, bilateral salpingoophphorectomy and excision of pelvic endometriosis. Histology from the right uterosacral ligament nodule showed fragments of an adenocarcinoma with a tubulopapillary architecture and hyalinised cores. The tumour cells were predominantly clear cells with some admixed eosinophilic cells, consistent with a clear cell carcinoma. There was no evidence of primary disease elsewhere within the gynaecological tract. The uterus was lined by proliferative endometrium and both ovaries contained foci of endometriosis but no evidence of atypia or carcinoma. There was extensive endometriosis throughout the remaining pelvic specimens but no further evidence of malignancy. The patient was referred to a tertiary centre for ongoing care and continues with this. Most recently she has suspected recurrent disease invading the right sacrum and pelvic nodes and is undergoing chemotherapy. Primary peritoneal clear cell carcinoma is rare, with only fourteen cases previously reported in the literature. Patients have been treated as per ovarian carcinoma with a variable response and it is considered to have a poor prognosis.

\section{IGCS20_1374}

\section{FACTORS ASSOCIATED WITH ACUTE KIDNEY INJURY IN PATIENTS UNDERGOING OPEN GYNECOLOGIC SURGERY ON AN ENHANCED RECOVERY AFTER SURGERY PATHWAY}

\begin{abstract}
${ }^{1} S$ Huepenbecker*, 'M Iniesta, 'P Ramirez, ${ }^{1} \mathrm{~B}$ Pitcher, ${ }^{2} \mathrm{M}$ Vaughn, ${ }^{3} \mathrm{G}$ Mena, ${ }^{3} \mathrm{~J}$ Lasala, ${ }^{1} \mathrm{~L}$ Meyer. ${ }^{1}$ Department of Gynecologic Oncology and Reproductive Medicine, The University of Texas MD Anderson Cancer Center, USA; ${ }^{2}$ The University of Texas Medical Branch, USA; ${ }^{3}$ Department of Anesthesiology and Perioperative Medicine, The University of Texas MD Anderson Cancer Center, USA
\end{abstract}

\subsection{6/ijgc-2020-IGCS.301}

Introduction Enhanced recovery after surgery (ERAS) pathways use goal-directed fluid therapy (GDT) to reduce postoperative complications from fluid imbalance. Our study aimed to determine the incidence and risks of acute kidney injury (AKI) in patients on an ERAS pathway.
Methods AKI was defined as acute risk, injury, or failure by RIFLE criteria. Chi-squared, Fisher's Exact, and Wilcoxon rank-sum tests were used. Propensity score analysis with 1:1 matching compared AKI in ERAS and pre-ERAS cohorts and modeled the probability of AKI as a function of ERAS.

Results Among 1127 patients on an ERAS pathway, 140 had AKI (12.4\%, 95\% CI 10.5\%-14.5\%). Patients with AKI were more likely to be older (median age 65 vs $57, \mathrm{p}<0.001$ ) and black $(18 \%$ vs $11 \%, \mathrm{p}=0.04)$ with more comorbidities (Charlson Comorbidity Index $\geq 3$ in $74 \%$ vs $48 \%, p<0.001$ ) including diabetes $(21 \%$ vs $12 \%, p=0.005)$, and had higher estimated blood loss (median 400 vs $250 \mathrm{~mL}, \mathrm{p}<0.001$ ), postoperative hypotension ( $6 \%$ vs $2 \%, \mathrm{p}=0.01)$, readmission $(20 \%$ vs $9 \%, \mathrm{p}<0.001)$, and reoperation $(5 \%$ vs $1.3 \%, \mathrm{p}=0.008)$ with longer median hospital stay ( 4 vs 3 days, $\mathrm{p}<0.001$ ). In 140 matched pairs, 9 pre-ERAS patients (6.4\%) and 17 ERAS patients $(12.1 \%)$ had AKI, with no effect of ERAS on AKI incidence (OR 0.5, p=0.11).

Conclusions A total of $12.4 \%$ of patients developed AKI, but ERAS itself was not associated with AKI incidence in the matched cohort. Highest AKI risk factors included older age, black race, more comorbidities, and blood loss.

Abstract 350 Table 1 Patient demographics and clinical characteristics associated with AKI within ERAS

\begin{tabular}{|c|c|c|c|}
\hline Variable & $\begin{array}{c}\text { no AKI } \\
\mathrm{N}=987\end{array}$ & $\begin{array}{c}\text { AKI } \\
\mathrm{N}=140\end{array}$ & P-value \\
\hline Age [median (min, max)] & $57.0(18.0,87.0)$ & $65.0(33.0,86.0)$ & $<0.001$ \\
\hline Charlson Comorbidity Index (CCI) & & & $<0.001$ \\
\hline 0 & $115(11.7 \%)$ & $3(2.1 \%)$ & \\
\hline $1-2$ & $400(40.5 \%)$ & $34(24.3 \%)$ & \\
\hline $3+$ & $472(47.8 \%)$ & $103(73.6 \%)$ & \\
\hline Comorbidity - Diabetes Mellitus (yes) & $121(12.3 \%)$ & $30(21.4 \%)$ & 0.005 \\
\hline BMI & & & $<0.001$ \\
\hline Normal & $631(66.9 \%)$ & $59(42.1 \%)$ & \\
\hline Obesity Class I & $163(16.5 \%)$ & $31(22.1 \%)$ & \\
\hline Obesity Class II & $82(8.3 \%)$ & $18(12.9 \%)$ & \\
\hline Obesity Class III & $92(9.3 \%)$ & $32(22.9 \%)$ & \\
\hline Unknown & $19(1.9 \%)$ & $0(0 \%)$ & \\
\hline Ethnicity & & & 0.548 \\
\hline Hispanic or Latino & $156(15.8 \%)$ & $25(17.9 \%)$ & \\
\hline Not Hispanic or Latino & $796(80.6 \%)$ & $108(77.1 \%)$ & \\
\hline Unknown & $35(3.5 \%)$ & $7(5.0 \%)$ & \\
\hline Race & & & 0.036 \\
\hline White or Caucasian & $682(69.1 \%)$ & $93(66.4 \%)$ & \\
\hline Black or African American & $106(10.7 \%)$ & $25(17.9 \%)$ & \\
\hline Asian & $61(6.2 \%)$ & $2(1.4 \%)$ & \\
\hline Native Hawaiian or Other Pacific Islander & $2(0.2 \%)$ & $0(0 \%)$ & \\
\hline American Indian or Alaskan Native & $2(0.2 \%)$ & $1(0.7 \%)$ & \\
\hline Unknown & $134(13.6 \%)$ & $19(13.6 \%)$ & \\
\hline Prior Chemo (yes) & $384(38.9 \%)$ & $58(41.4 \%)$ & 0.567 \\
\hline Prior Radiation (yes) & $23(2.3 \%)$ & $5(3.6 \%)$ & 0.378 \\
\hline Tumor Type & & & $<0.001$ \\
\hline Benign & $209(21.2 \%)$ & $15(10.7 \%)$ & \\
\hline Malignant & $672(68.1 \%)$ & $117(83.6 \%)$ & \\
\hline Recurrent & $106(10.7 \%)$ & $8(5.7 \%)$ & \\
\hline Organ Site & & & $<0.001$ \\
\hline Cervix & $42(4.2 \%)$ & $3(2.1 \%)$ & \\
\hline Uterine & $127(12.9 \%)$ & $39(27.9 \%)$ & \\
\hline Fallopian Tube, Ovary, Peritoneum & $503(51.0 \%)$ & $75(53.6 \%)$ & \\
\hline Benign & $209(21.2 \%)$ & $15(10.7 \%)$ & \\
\hline Recurrent & $106(10.7 \%)$ & $\begin{array}{l}8(5.7 \%) \\
-15\end{array}$ & \\
\hline Preop Hemoglobin [median (min, max)] & $12.0(6.3,16.4)$ & $11.5(7.1,15.8)$ & 0.002 \\
\hline Preop creatinine [median (min, max)] & $0.7(0.4,10.4)$ & $0.8(0.5,1.7)$ & $<0.001$ \\
\hline Preop creatinine clearance [median (min, max)] & $96.1(8.5,299.2)$ & $89.3(30.6,217.6)$ & 0.288 \\
\hline Estimated Blood Loss ( $\mathrm{ml}$ [median (min, max)] & $250(5,5550)$ & $400(20,4000)$ & $<0.001$ \\
\hline OR Time (minutes) [median (min, max)] & $211(33,1437)$ & $263(58,686)$ & $<0.001$ \\
\hline IV Tylenol (yes) & $509(51.6 \%)$ & $55(39.3 \%)$ & 0.007 \\
\hline Goal-directed therapy (yes) & $560(56.8 \%)$ & $89(63.6 \%)$ & 0.144 \\
\hline Crystalloids (mL) [median (min, max)] & $1500(0,4510)$ & $1700(350,6600)$ & $<0.001$ \\
\hline Colloids (mL) [median (min, max)] & $500(25,3000)$ & $1000(250,2500)$ & $<0.001$ \\
\hline Net Fluid Balance $(\mathrm{mL})[$ median (min, $\mathrm{max})]$ & $1250(-6420,618)$ & $1515(-936,7170)$ & $<0.001$ \\
\hline Length of stay (days) [median (min, max)] & $3(1,43)$ & $4(2,57)$ & $<0.001$ \\
\hline Readmission & $92(9.3 \%)$ & $28(20.0 \%)$ & $<0.001$ \\
\hline Reoperation & $13(1.3 \%)$ & $7(5.0 \%)$ & 0.008 \\
\hline Hypotension & $18(1.8 \%)$ & $8(5.7 \%)$ & 0.010 \\
\hline Hypertension & $16(1.6 \%)$ & $6(4.3 \%)$ & 0.045 \\
\hline Any Grade 1-2 complications & $527(53.4 \%)$ & $140(100.0 \%)$ & $<0.001$ \\
\hline Any Grade $3+$ complications & $59(6.0 \%)$ & $33(23.6 \%)$ & $<0.001$ \\
\hline
\end{tabular}

